\title{
A helical, aromatic, peptide nanotube
}

\author{
Marco Crisma, ${ }^{*}, \uparrow$ Claudio Toniolo, ${ }^{\dagger}$ Soledad Royo, ${ }^{\ddagger}$ Ana I. Jiménez, ${ }^{\ddagger}$ and Carlos Cativiela ${ }^{*}$, \\ ${ }^{\ddagger}$ Department of Organic Chemistry, ICMA, University of Zaragoza-CSIC, 50009 Zaragoza (Spain) \\ ${ }^{\dagger}$ Institute of Biomolecular Chemistry, CNR, Department of Chemistry, University of Padova, 35131 Padova (Italy)
}

Supporting Information

\section{General remarks:}

NMR spectra of peptide 1 were recorded on a Bruker Avance DRX 400 spectrometer at room temperature, using the residual non-deuterated solvent signal as the internal standard; chemical shifts $(\delta)$ are expressed in ppm and coupling constants $(J)$ in Hertz. Peak assignment was made according to the information provided by COSY, HSQC and HMBC experiments. The IR spectrum was registered on a Mattson Genesis FTIR spectrophotometer; $v_{\max }$ is given for the main absorption bands. The optical rotation was measured at room temperature using a JASCO P-1020 polarimeter. The high-resolution mass spectrum was obtained on a Bruker Microtof-Q spectrometer. The melting point was determined on a Gallenkamp apparatus. The preparation and characterization of compounds Boc- $(S, S) \mathrm{c}_{3} \mathrm{diPhe}-\mathrm{OH}^{\mathrm{S1}}$ and Boc$(R, R) \mathrm{c}_{3}$ diPhe- $\mathrm{NH}^{i} \mathrm{Pr}^{\mathrm{S} 2}$ were already reported.

Synthesis of Boc- $(\boldsymbol{S}, S) \mathbf{c}_{3}$ diPhe- $(\boldsymbol{R}, \boldsymbol{R}) \mathbf{c}_{3}$ diPhe-NH ${ }^{i} \operatorname{Pr}(\mathbf{1})$. To a solution of Boc- $(S, S) \mathrm{c}_{3}$ diPhe-OH (304 mg, $\left.0.86 \mathrm{mmol}\right)$ in dry $\mathrm{CH}_{2} \mathrm{Cl}_{2}(4 \mathrm{~mL})$ at $0^{\circ} \mathrm{C}$, HOAt $(59 \mathrm{mg}, 0.43 \mathrm{mmol}), \mathrm{HATU}^{\mathrm{S3}}(327 \mathrm{mg}, 0.86 \mathrm{mmol})$ and DIEA $(0.57 \mathrm{~mL}, 3.44$ mmol) were added. After $10 \mathrm{~min}, \mathrm{HCl} \cdot \mathrm{H}-(R, R) \mathrm{c}_{3} \mathrm{diPhe}-\mathrm{NH}^{i} \mathrm{Pr}$ [obtained by treatment of the corresponding Bocprotected amino acyl isopropylamide (355 $\mathrm{mg}, 0.90 \mathrm{mmol}$ ) with a $3 \mathrm{~N}$ solution of $\mathrm{HCl}$ in dry EtOAc] was added. The reaction mixture was stirred at room temperature for 4 days and then evaporated in vacuo. The residue was dissolved in EtOAc $(300 \mathrm{~mL})$ and the solution was washed with $10 \% \mathrm{KHSO}_{4}(3 \times 100 \mathrm{~mL})$, water $(2 \times 100 \mathrm{~mL}), 5 \% \mathrm{NaHCO}_{3}(2 \times 100$ $\mathrm{mL})$, water $(2 \times 100 \mathrm{~mL})$, dried over anhydrous $\mathrm{Na}_{2} \mathrm{SO}_{4}$, filtered and evaporated to dryness. Recrystallization of the crude product from $\mathrm{Et}_{2} \mathrm{O} / \mathrm{PE}$ afforded the title compound $(489 \mathrm{mg}, 0.78 \mathrm{mmol}, 90 \%$ yield) as a white solid.

mp: $122-123^{\circ} \mathrm{C}\left(\mathrm{Et}_{2} \mathrm{O} / \mathrm{PE}\right)$

$[\alpha]^{26}$ : $-65.6(c 0.50, \mathrm{MeOH})$

$R_{f}: 0.69\left(\mathrm{CH}_{2} \mathrm{Cl}_{2} / \mathrm{EtOAc} 8 / 2\right)$

IR (nujol): v 3420, 3368, 1700, $1643 \mathrm{~cm}^{-1}$

${ }^{1} \mathrm{H}$ NMR $\left(400 \mathrm{MHz}, \mathrm{CDCl}_{3}\right): \delta 0.87\left(\mathrm{~d}, J=6.4 \mathrm{~Hz}, 3 \mathrm{H},{ }^{i} \operatorname{Pr~} \mathrm{CH}_{3}\right), 1.02\left(\mathrm{~d}, J=6.4 \mathrm{~Hz}, 3 \mathrm{H},{ }^{i} \mathrm{Pr} \mathrm{CH}_{3}\right), 1.26$ (s, 9H, Boc $\left.\mathrm{CH}_{3}\right), 2.44\left(\mathrm{~d}, J=8.6 \mathrm{~Hz}, 1 \mathrm{H}, \mathrm{c}_{3} \mathrm{diPhe} \mathrm{H}^{\beta}\right), 3.03\left(\mathrm{~d}, J=8.4 \mathrm{~Hz}, 1 \mathrm{H}, \mathrm{c}_{3} \mathrm{diPhe} \mathrm{H}^{\beta}\right), 3.72\left(\mathrm{~d}, J=8.6 \mathrm{~Hz}, 1 \mathrm{H}, \mathrm{c}_{3} \mathrm{diPhe} \mathrm{H}^{\beta}\right)$, $3.79\left(\mathrm{~m}, 1 \mathrm{H},{ }^{i} \mathrm{Pr} \mathrm{CH}\right), 3.99$ (br d, $J=8.4 \mathrm{~Hz}, 1 \mathrm{H}, \mathrm{c}_{3} \mathrm{diPhe} \mathrm{H}^{\beta}$ ), 4.64 (br s, $1 \mathrm{H}, \mathrm{c}_{3}$ diPhe NH), 6.49 (br s, $1 \mathrm{H}, \mathrm{c}_{3} \mathrm{diPhe} \mathrm{NH}$ ), $6.85\left(\mathrm{~m}, 1 \mathrm{H}, \mathrm{NH}^{i} \mathrm{Pr}\right), 7.10-7.50(\mathrm{~m}, 20 \mathrm{H}, \mathrm{Ar})$.

${ }^{13} \mathrm{C}$ NMR $\left(100 \mathrm{MHz}, \mathrm{CDCl}_{3}\right): \delta 22.29\left({ }^{i} \mathrm{Pr} \mathrm{CH}_{3}\right), 22.37\left({ }^{i} \mathrm{Pr} \mathrm{CH}_{3}\right), 28.07\left(\mathrm{Boc} \mathrm{CH}_{3}\right), 32.70\left(\mathrm{c}_{3} \mathrm{diPhe} \mathrm{C}^{\beta}\right), 33.34\left(\mathrm{c}_{3} \mathrm{diPhe}\right.$ $\left.\mathrm{C}^{\beta}\right), 37.35\left(\mathrm{c}_{3} \mathrm{diPhe} \mathrm{C}^{\beta}\right), 38.53\left(\mathrm{c}_{3} \mathrm{diPhe} \mathrm{C}^{\beta}\right), 41.65\left({ }^{i} \mathrm{Pr} \mathrm{CH}\right), 46.63\left(\mathrm{c}_{3} \mathrm{diPhe} \mathrm{C}^{\alpha}\right), 48.54\left(\mathrm{c}_{3} \mathrm{diPhe} \mathrm{C}^{\alpha}\right), 80.91$ (Boc C), $126.70,127.23,127.27,127.64,127.86,128.23,128.48,128.57,128.98,129.00,129.09,129.15,134.11,134.35$, 134.88, 135.60 (Ar), 156.07 (Boc CO), 166.46 ( $\mathrm{c}_{3}$ diPhe CO), 169.65 ( $\mathrm{c}_{3}$ diPhe CO).

HRMS (ESI) $\mathrm{C}_{40} \mathrm{H}_{44} \mathrm{~N}_{3} \mathrm{O}_{4}[\mathrm{M}+\mathrm{H}]^{+}$: calcd 630.332633, found 630.333899.

\section{Abbreviations}

Boc, tert-butyloxycarbonyl; DIEA, $N, N$-diisopropylethylamine; HATU, $\{N$-[(dimethylamino)-1H-1,2,3-triazolo[4,5- $b]$ pyridin-1-yl-methylene $]-N$ methylmethanaminium hexafluorophosphate $N$-oxide $;$; HOAt, 1-hydroxy-7-azabenzotriazole; PE, petroleum ether; ${ }^{i} \mathrm{Pr}$, isopropyl.

\section{References}

(S1) Jiménez, A. I.; López, P.; Oliveros, L.; Cativiela, C. Tetrahedron 2001, 57, 6019-6026.

(S2) Royo, S.; De Borggraeve, W. M.; Peggion, C.; Formaggio, F.; Crisma, M.; Jiménez, A. I.; Cativiela, C.; Toniolo, C. J. Am. Chem. Soc. 2005, 127, 2036-2037.

(S3) Carpino, L. A. J. Am. Chem. Soc. 1993, 115, 4397-4398. 


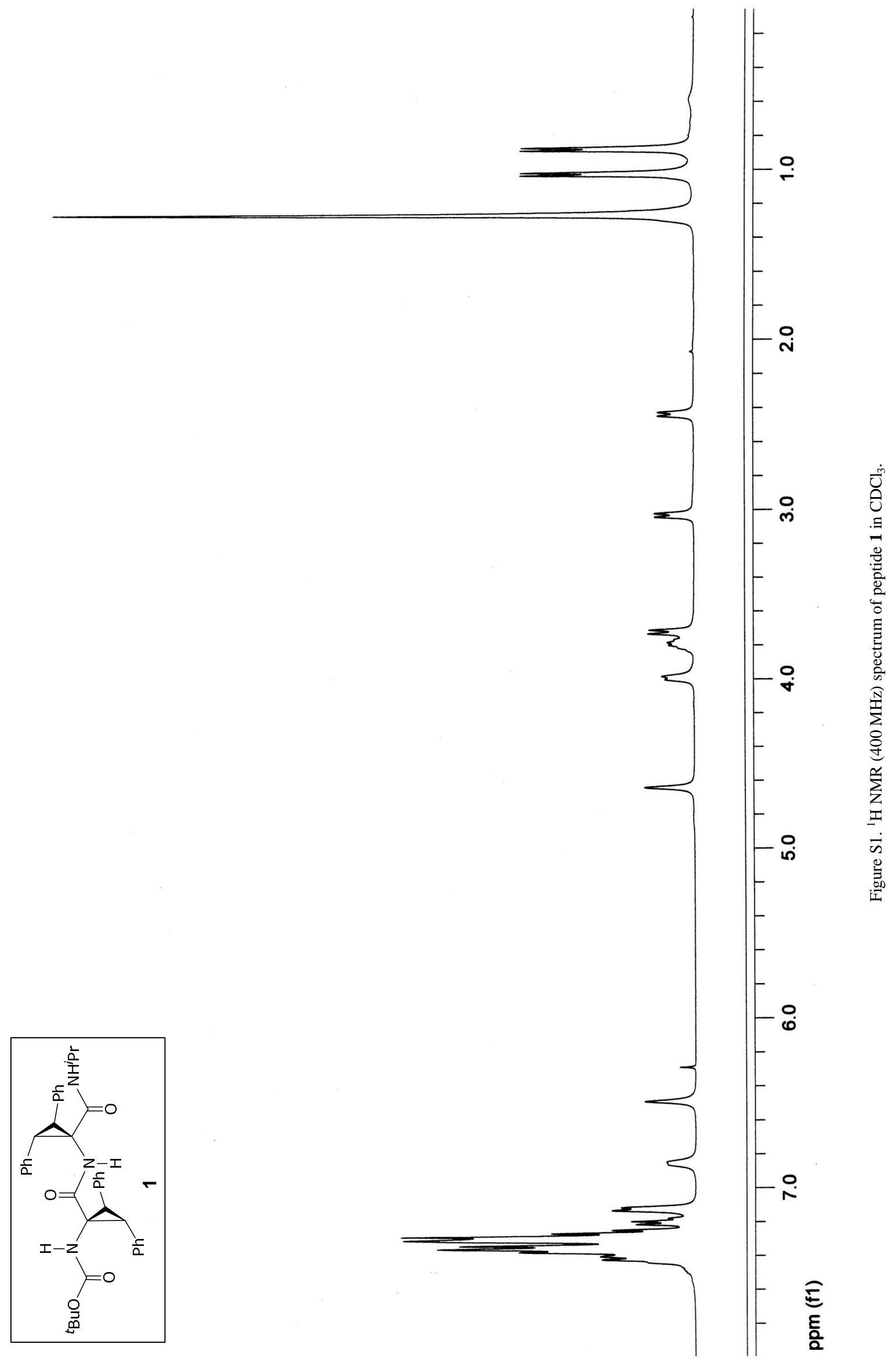




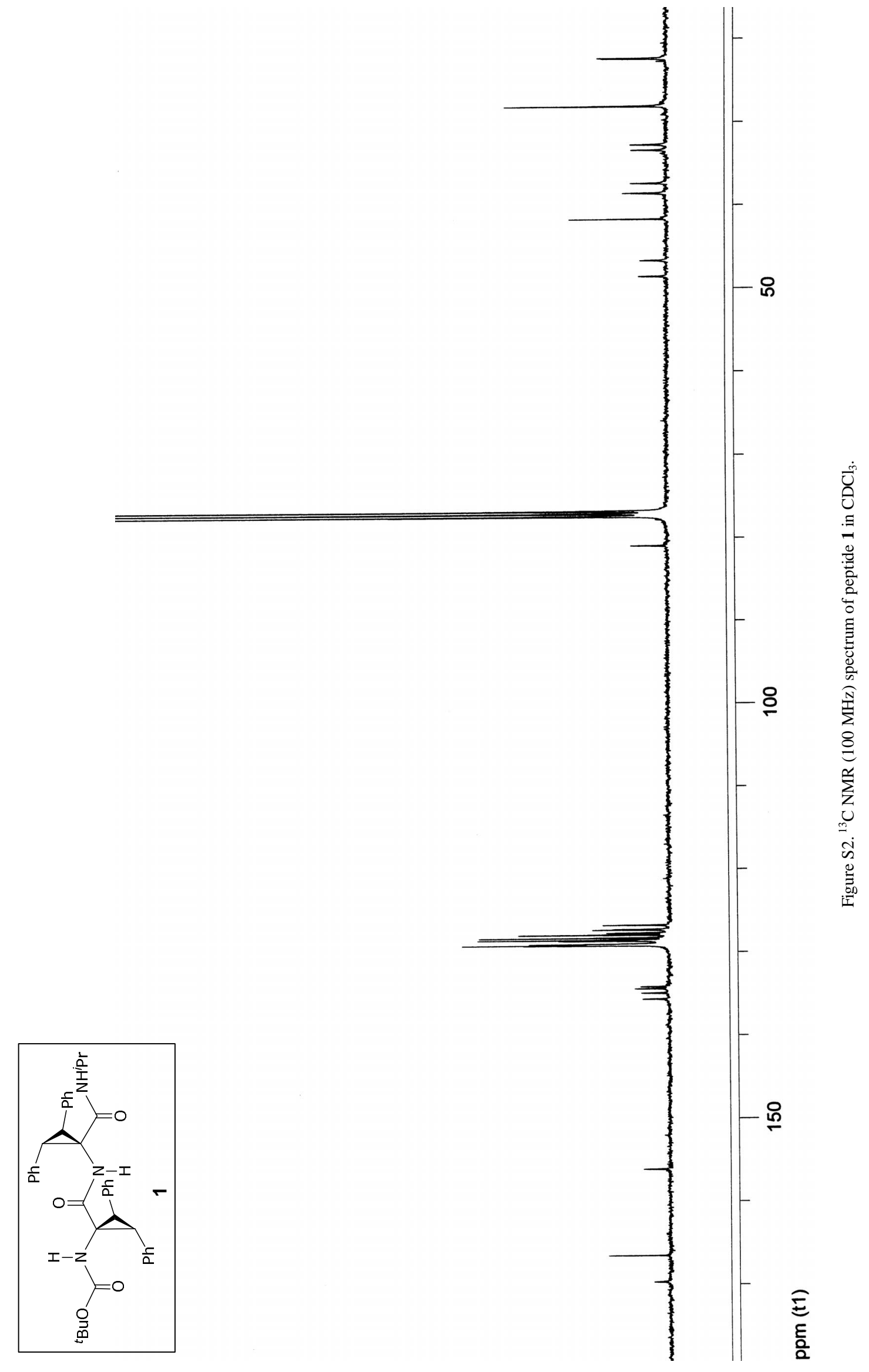

